Article

\title{
New Instrument for Oral Hygiene of Children with Cleft Lip and Palate
}

\author{
Rita Rodrigues 1,2 (1), Maria Helena Fernandes 1,3 (-) , António Bessa Monteiro ${ }^{4}$, \\ Rowney Furfuro ${ }^{4}$, Cátia Carvalho Silva ${ }^{2}$, César Soares ${ }^{5}$ (i), Ricardo Vardasca ${ }^{5}$ (i),

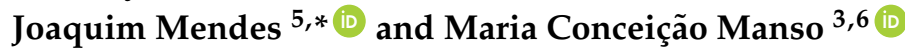 \\ 1 Faculty of Dentistry, University of Porto, R. Dr. Manuel Pereira da Silva, 93, 4200-393 Porto, Portugal; \\ carlarmendes@hotmail.com (R.R.); mhfernandes@fmd.up.pt (M.H.F.) \\ 2 Faculty of Health Sciences, University Fernando Pessoa, Praça 9 de Abril, 349, 4249-004 Porto, Portugal; \\ catiasilva@ufp.edu.pt \\ 3 Associated Laboratory for Green Chemistry (LAQV/REQUIMTE), University of Porto, \\ 4051-401 Porto, Portugal; cmanso@ufp.edu.pt \\ 4 Faculty of Medicine, University of Porto, Alameda Prof. Hernâni Monteiro, s/n, 4200-319 Porto, Portugal; \\ bessamonteiro@gmail.com (A.B.M.); rowney.furfuro@gmail.com (R.F.) \\ 5 Faculty of Engineering, University of Porto, R. Dr. Roberto Frias, s/n, 4200-465 Porto, Portugal; \\ em04018@fe.up.pt (C.S.); ricardo.vardasca@fe.up.pt (R.V.) \\ 6 Faculty of Health Sciences, University Fernando Pessoa, Fernando Pessoa Energy, \\ Environment and Health Research Unit (FP-ENAS), Praça de 9 de Abril, 349, 4249-004 Porto, Portugal \\ * Correspondence: jgabriel@fe.up.pt; Tel.: +351-220-413-411
}

Received: 5 March 2018; Accepted: 5 April 2018; Published: 19 April 2018

\begin{abstract}
Patients with cleft lip and palate (CLP) have several oral alterations that make the removal of bacterial plaque in the cleft area difficult, which is the main cause of the high incidence of oral health problems. The aim of this study is to develop a toothbrush especially designed to perform the hygiene of the cleft area. This device, called Cleft Toothbrush, is an add-on to a conventional toothbrush to clean hard-to-reach areas that are not accessible by using conventional brush shapes. The design was made in SOLIDWORKS $®$ (Waltham, MA, USA) 3D and a sample of this device was produced by CURAPROX (Kriens, Switzerland). The efficacy of the Cleft Toothbrush was tested in a preliminary study performed in a four years old child with CLP, compared to that of a conventional toothbrush. A significantly higher reduction of the bacterial plaque was achieved with the Cleft Toothbrush. The child's parents also felt more confident with this device, as it deals better with sensitive areas, as it did not hurt the child. This innovative toothbrush adapter appears to greatly improve the cleaning areas of high bacterial plaque retention in patients with CLP, together with excellent acceptance.
\end{abstract}

Keywords: cleft lip and palate; finite element analysis; oral hygiene; toothbrush

\section{Introduction}

Cleft lip and palate (CLP) are the most common congenital malformations of the face that may appear isolated, or in association with other syndromes. They result from the failure of the fusion of the many embrionic processes, between the 4th and 12th weeks of intrauterine life. Clinically, the anatomic-functional alteration of one, or many, structures (lip, alveolar process, hard and soft palate) is observed. The cleft lip, or cleft palate, can occur alone or together, and might be complete or incomplete, uni- or bilateral [1,2].

The morphology of the space of the cleft in the oral cavity, although variable, presents common elements. It is frequently originated by the collapse of the lateral segments of the maxilla towards the 
medial sagittal plane, the ends of the alveolar ridges creating a gap of an approximately triangular shape, with vertex towards occlusal and the base of the triangle towards to the cleft. This triangular shape is similar to the shape of the niches (Figure 1).

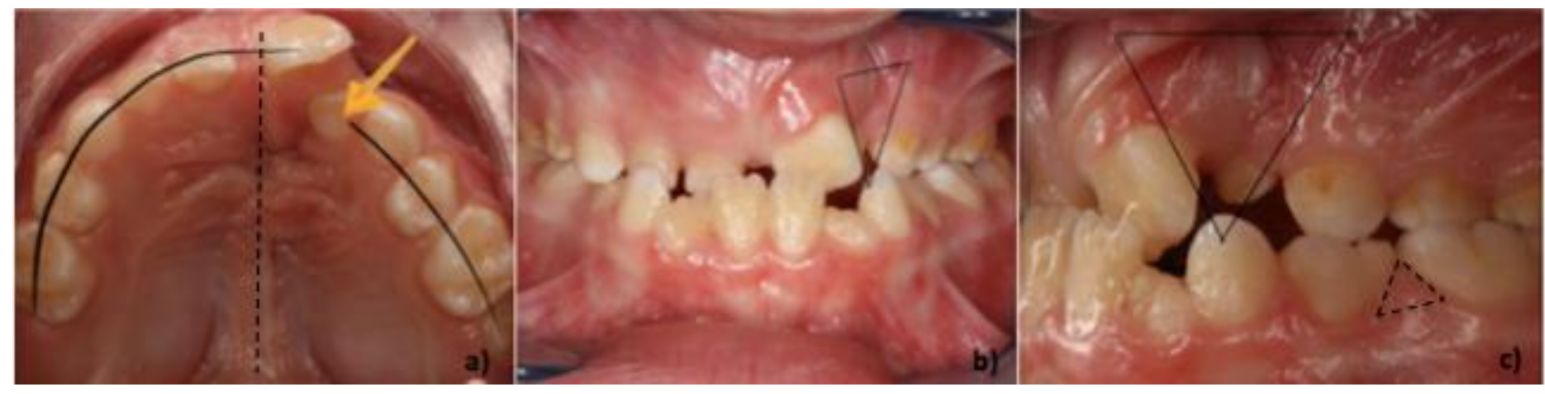

Figure 1. Intraoral photographs of a child with cleft lip and palate, Spina type II (complete cleft lip and palate on the left side): (a) collapse of the lateral segments of the maxilla (dashed line, medial sagittal plane), the arrow shows a supernumerary tooth in the cleft area; (b) frontal photograph and (c) left lateral photograph-space of triangular shape, with vertex towards occlusal and the base of the triangle towards to the cleft; and (c) the triangular shape of the cleft is similar to those of the tooth niches (dashed triangle).

Bone and dental changes in children with CLP are associated with a high incidence of oral health problems [3-5]. The anatomical and morphological alterations increase the accumulation of bacterial plaque and, further, increase the difficulty of its removal. Thus, the high prevalence of dental caries in these children is well documented [6-10]. Although preventive measures related to dietary counseling and the use of antiseptic mouthwash help to minimize the problem, there is no substitute for good toothbrushing to effectively remove dental plaque [11].

The relevance of cleaning the cleft area in order to maintain good oral health is highlighted in a variety of studies $[4,8,11,12]$. However, a several reasons account for the difficulties of children and parents in performing an appropriate toothbrushing cleaning, mainly fear of soft tissue trauma during brushing, worries about bleeding from inflamed gingiva, and difficult access to the teeth and the deepest area of the cleft $[8,13]$. Even so, little attention has been given to a key issue-the toothbrush. We found only one study that refers that the use of a baby-sized toothbrush and an interspace brush would improve the cleft hygiene in some situations [11].

In this context, there is a clear need to develop improved methods of accessing and cleaning areas of high retention of the biofilm, either by the cleft morphology or the sensitivity of the surrounding tissues due to frequent local inflammatory conditions.

The aim of this study is then to develop a toothbrush especially designed to improve the hygiene of the cleft area.

\section{Design of the Cleft Toothbrush}

Given the morphology of the cleft, the design of a new hygiene tool should take into account aspects such as adaptability to the contour of the cleft and brushing material. Additionally, assuming children will mostly use this tool, the degree of difficulty of use should be the smallest possible.

As interdental brushes are usually used for better hygiene between teeth, the design of the Cleft Toothbrush started from a common interdental brush, which was then adapted to meet the specificities of a larger and more complex space. While the brush could perform its function with simple back-and-forth movements, the new brush has to be different. The semi-circular movement of rotation in relation to its own axis was found to be the one that shows the best results. Thus, the design of the Cleft Toothbrush became that of a flame shape, as to better fit the space. The use of $360^{\circ}$ bristles 
allows the user to act on the brush handle with wide movements and produce a friction effect by rotation, without causing trauma to the mucous membranes.

The angulation between the handle and the tip was designed to facilitate the approach and access to the gap. With this small angle, the rotation of the brush is obtained not by the rotation of the handle on its own longitudinal axis, but by the semi-circumferential movement of the handle. This movement is very similar to brushing with a conventional brush, a factor of greater adhesion and ease of implementation of the oral hygiene routine. This tip acts as a spring, making it easier to maintain a contact force during brushing, its diameter being small enough to facilitate access to the cleft.

The bristles cannot be long as they would be too flexible and would not cause enough interference to remove the plaque. On the other hand, they should not be too short, as they would not have the desired flexibility and would hurt the surrounding cleft tissues. The solution was to implement bristles of different lengths along the axis, allowing a better docking of the new instrument to the cleft, increasing the contact with the zone to be cleaned, thus leading to a greater removal of existing bacterial plaque along the cleft zone.

Based on these ideas and requirements, it was designed in SOLIDWORKS®and the 3D model shown in Figure 2a will be analyzed in detail in the next section.

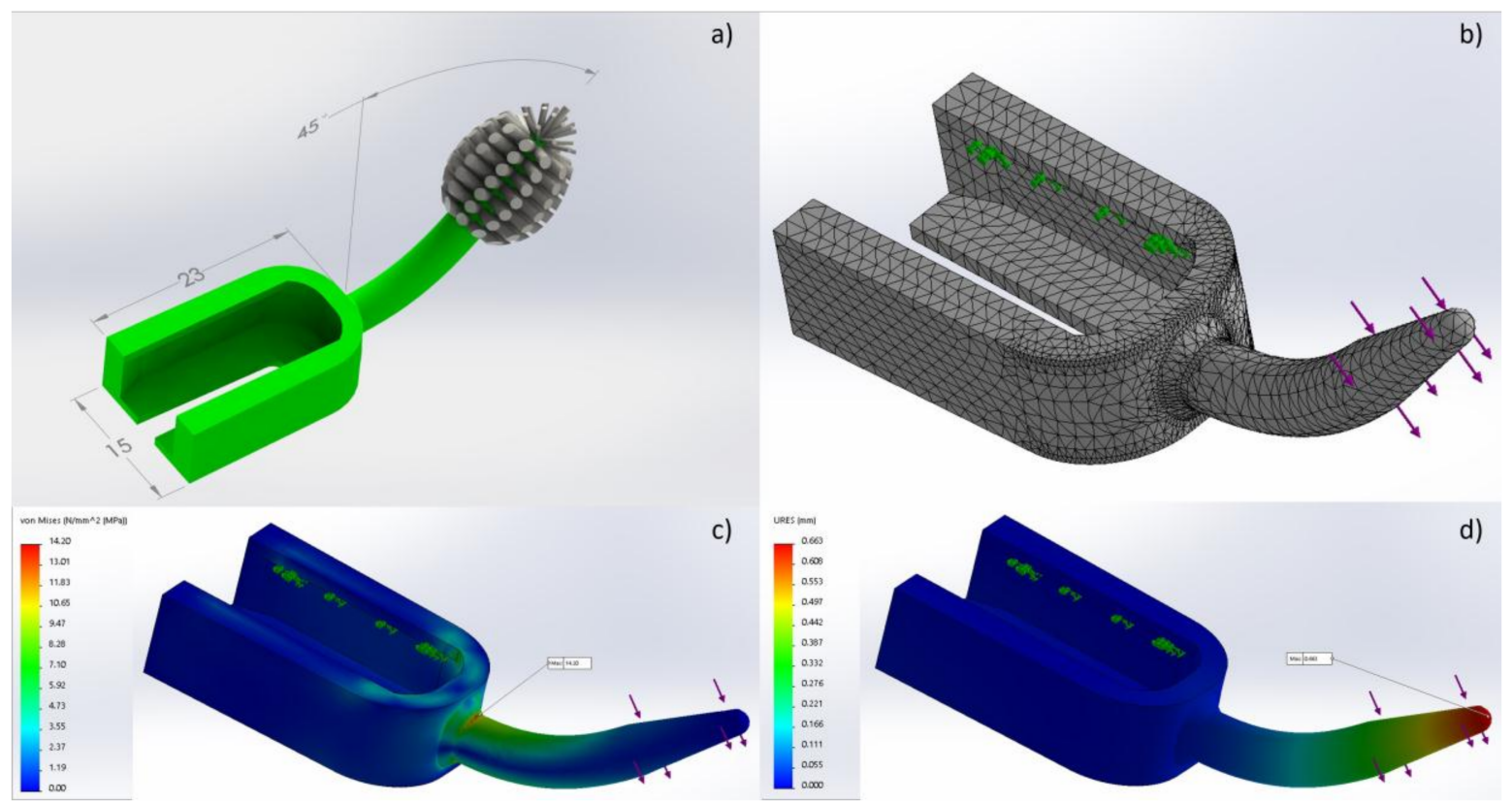

Figure 2. (a) Cleft Toothbrush-3D model; (b) finite elements solid mesh; (c) results of the stress in the Cleft Toothbrush due to the applied load (maximum of $14.20 \mathrm{MPa}$ at the connection between the horn and the body); and (d) the maximum displacement of the adapter due to the applied load- $0.663 \mathrm{~mm}$ reached in the tip (red area).

\section{Finite Element Simulation}

Finite element analysis (FEA) was applied to the Cleft Toothbrush 3D model in order to validate the mechanical strength. For this purpose, a force of $3 \mathrm{~N}$ [14-16] was applied to the tip of the "horn"-like zone, perpendicularly to its axis. The material chosen for the body of the toothbrush was acrylonitrile-butadiene-styrene (ABS) as it assures a good surface finish, and impact and tensile strength (30 MPa).

The simulation was made in SOLIDWORKS®Simulation, assuming a non-linear behavior of the material. The mesh was generated using a solid structure with variable size, with 26,000 nodes, Figure $2 b, c$ presents the toothbrush model simulation, where colors represent the mechanical stress 
level achieved while applying a force of $3 \mathrm{~N}$. It can be seen that the most stressed site is the connection between the two parts (the body of the adapter and the "horn"-like zone), corresponding to the red area. However, it still about half of the maximum stress that the material can withstand, so it is perfectly safe to be used. Figure $2 \mathrm{~d}$ shows the displacement of the model surface as a consequence of the applied force, which will be about $0.7 \mathrm{~mm}$ at the tip, corresponding to the red color.

\section{The Efficacy of the Cleft Toothbrush}

A prototype of the Cleft Toothbrush was fabricated by the company CURAPROX, using a 3D fused deposition modelling (FDM) technique. The body was built in ABS, while the bristles were made of nylon.

The efficacy of the Cleft Toothbrush was evaluated in a preliminary study performed in a child with a cleft lip and palate, Spina classification type I [17], four year old, male, after informed consent by his parents. The study was conducted in accordance with the Declaration of Helsinki, and the protocol was approved by the Ethics Committee of the Faculty of Dentistry, University of Porto, Portugal.

The performance of the Cleft Toothbrush in the bacterial plaque removal was compared with that of a manual conventional toothbrush (small head brush, bristles of medium hardness, all of the same size, which were present only in one side of the brush), using the same protocol.

Due to the child's age, his parents were responsible for his oral hygiene, in accordance with the American Academy of Pediatric Dentistry [18]. The study was performed at the participant's home using the protocol below.

First, the clef area to be evaluated was clearly defined. This included the vestibular faces of the adjacent teeth, on the left (supernumerary canine) and right side (central incisor) (Figure 3a).

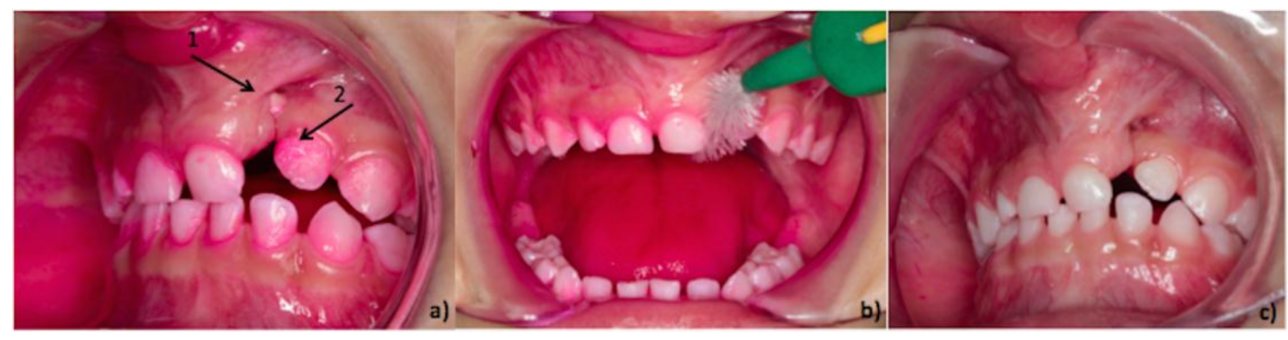

Figure 3. Intraoral photographs from a child with cleft lip and palate, Spina type I, (a complete cleft lip on the left side); (a) the teeth were stained to evidence the bacterial plaque, 1 - food in the deep cleft, 2-The supernumerary is the tooth with more bacterial plaque on the surface; (b) brushing with the Cleft Toothbrush - the front bristles were used to enter the cavity of the cleft, combining rotation and translation movements; and (c) the mouth after brushing, showing the high efficacy in bacterial plaque removal.

After, evaluation of the bacterial plaque was performed by using an Adapted Plaque Index to the Cleft (adapted PI-C) suitably adapted to be conducted at the child's home. First, in order to evidence the bacterial plaque, $2 \%$ erythrosine was used, as in the Greene and Vermillion index [19]. Then, the Adapted Plaque Index was calculated by dividing the sum of the amount of dental surface stained of each tooth by the number of observed teeth to obtain an average value. The adapted PI-C can have a score ranging from 0 to 3 , which corresponds to the amount of dental surface stained with erythrosine $2 \%$ ( 0 : no staining; 1 : staining $<1 / 3 ; 2$ : staining between $1 / 3$ and $2 / 3 ; 3$ : staining $>2 / 3$.

An adapted PI-C of 3 was selected as the baseline value for evaluating the efficacy of the Cleft Toothbrush in bacterial plaque removal, as well as for the conventional toothbrush, for comparative purposes.

The brushing procedure was conducted with either the Cleft Toothbrush or the conventional toothbrush using a well-defined protocol: brushing after breakfast and dinner, with a brushing duration of $2 \mathrm{~min}$ [20]. The brushing with the Cleft Toothbrush or the conventional toothbrush was performed in 
two different moments, i.e., separated by two weeks. Figure $3 b$ illustrates the cleft cleaning procedure using the designed Cleft Toothbrush.

Immediately after the last brushing procedure (after dinner), the plaque index was again evaluated, using the Adapted PI-C, in order to analyze the efficacy of the bacterial plaque removal. Using the Cleft Toothbrush, the Adapted PI-C decreased from 3 to 1, whereas with the conventional brush a reduction from 3 to 2 was achieved.

Additionally, the Cleft Toothbrush had a very good acceptance by the child and his parents. It was stated that the new cleft toothbrush was more effective in brushing and cleaning deeper areas of the cleft, compared to the conventional toothbrush. They also felt that the new brush was safer to use in the sensitive tissues of the cleft area.

\section{Discussion}

In the design of the brush, it was decided not to develop a complete brush from scratch, but instead to build an adapter that could be easily used in conjunction with CURAPROX brushes. This approach was intended to facilitate transport and lower the cost of the brush, as the wear and tear of the bristles of the brush and cleft brush would be different. Thus, by decoupling both, it allows the optimal use of each of the parts in a different way, maintaining the effectiveness of both, for the time that each one is effective.

The motivation for the design of this brush was based on a clinical need, verified by the constant presence of a high bacterial plaque index in the teeth adjacent to the slits. The developed Cleft Toothbrush has features that facilitate the cleansing of the cleft, as well as increase the child's comfort for the maintenance of the healthy tissues adjacent to the cleft. This device can be easily used by children either helped or not by adults, thus contributing to better oral hygiene.

Due to the encouraging results of this preliminary study, a large group size of children with CLP is now testing the Cleft Toothbrush to fully prove the present results that clearly point in the direction of an effective significant reduction of bacterial plaque, as well as acceptance and confidence. The authors believe that this brush adapter will certainly be of good help to the hygiene and health of these children.

\section{Patent}

A patent request was issued on the design of this toothbrush.

Acknowledgments: Maria Conceição Manso acknowledges Fundação para a Ciência e a Tecnologia through grant UID/Multi/04546/2013 and UID/ QUI/50006/2013-POCI/01/0145/FERDER/007265.

Author Contributions: The authors Rowney Furfuro, António Bessa Monteiro, and Rita Rodrigues conceived and designed the toothbrush; Joaquim Mendes, César Soares, and Ricardo Vardasca, conducted the computer design and validation using the finite element method; Maria Conceição Manso analyzed the data; and Rita Rodrigues, Joaquim Mendes, Maria Helena Fernandes, and Cátia Carvalho Silva wrote the paper.

Conflicts of Interest: The authors declare no conflict of interest.

\section{References}

1. Maarse, W.; Rozendaal, A.M.; Pajkrt, E.; Vermeij-Keers, C.; van der Molen, A.B.M.; van den Boogaard, M.-J.H. A systematic review of associated structural and chromosomal defects in oral clefts: When is prenatal genetic analysis indicated? J. Med. Genet. 2012, 49, 490-498. [CrossRef] [PubMed]

2. Sadler, T.W.T.W.; Langman, J.M.E. Langman's Medical Embryology, 12th ed.; Sadler, T.W., Ed.; Wolters Kluwer Health: Philadelphia, PA, USA; Lippincott Williams \& Wilkins: Philadelphia, PA, USA, 2012.

3. Lages, E.M.B.; Marcos, B.; Pordeus, I.A. Oral health of individuals with cleft lip, cleft palate, or both. Cleft Palate-Craniofac. J. 2004, 41, 59-63. [CrossRef] [PubMed]

4. Hazza'a, A.M.; Rawashdeh, M.A.; Al-Nimri, K.; Al Habashneh, R. Dental and oral hygiene status in jordanian children with cleft lip and palate: A comparison between unilateral and bilateral clefts. Int. J. Dent. Hyg. 2011, 9, 30-36. [CrossRef] [PubMed] 
5. Perdikogianni, H.; Papaioannou, W.; Nakou, M.; Oulis, C.; Papagiannoulis, L. Periodontal and microbiological parameters in children and adolescents with cleft lip and/or palate. Int. J. Paediatr. Dent. 2009, 19, 455-467. [CrossRef] [PubMed]

6. Shashni, R.; Goyal, A.; Gauba, K.; Utreja, A.K.; Ray, P.; Jena, A.K. Comparison of risk indicators of dental caries in children with and without cleft lip and palate deformities. Contemp. Clin. Dent. 2015, 6, 58-62. [PubMed]

7. Worth, V.; Perry, R.; Ireland, T.; Wills, A.K.; Sandy, J.; Ness, A. Are people with an orofacial cleft at a higher risk of dental caries? A systematic review and meta-analysis. Br. Dent. J. 2017, 223, 37-47. [CrossRef] [PubMed]

8. Ahluwalia, M.; Brailsford, S.R.; Tarelli, E.; Gilbert, S.C.; Clark, D.T.; Barnard, K.; Beighton, D. Dental caries, oral hygiene, and oral clearance in children with craniofacial disorders. J. Dent. Res. 2004, 83, 175-179. [CrossRef] [PubMed]

9. Antonarakis, G.; Palaska, P.-K.; Herzog, G. Caries prevalence in non-syndromic patients with cleft lip and/or palate: A meta-analysis. Caries Res. 2013, 47, 406-413. [CrossRef] [PubMed]

10. Cheng, L.L.; Moor, S.L.; Ho, C.T. Predisposing factors to dental caries in children with cleft lip and palate: A review and strategies for early prevention. Cleft Palate-Craniofac. J. 2007, 44, 67-72. [CrossRef] [PubMed]

11. Rivkin, C.J.; Keith, O.; Crawford, P.J.; Hathorn, I.S. Dental care for the patient with a cleft lip and palate. Part 2: The mixed dentition stage through to adolescence and young adulthood. Br. Dent. J. 2000, 188, 131-134. [CrossRef] [PubMed]

12. Lin, Y.L.; Davies, K.; Callery, P. Experience of maintaining tooth brushing for children born with a cleft lip and/or palate. BMC Oral Health 2017, 17, 120. [CrossRef] [PubMed]

13. Gopakumar, M.; Hegde, A.M. Parental attitude towards the provision of nonsurgical oral health care to children with oral clefts: An epidemiological survey. Int. J. Clin. Pediatr. Dent. 2010, 3, 35-37. [CrossRef] [PubMed]

14. van der Weijden, G.A.; Timmerman, M.F.; Reijerse, E.; Snoek, C.M.; van der Velden, U. Toothbrushing force in relation to plaque removal. J. Clin. Periodontol. 1996, 23, 724-729. [CrossRef] [PubMed]

15. Hayasaki, H.; Saitoh, I.; Nakakura-Ohshima, K.; Hanasaki, M.; Nogami, Y.; Nakajima, T.; Inada, E.; Iwasaki, T.; Iwase, Y.; Sawami, T.; et al. Tooth brushing for oral prophylaxis. Jpn. Dent. Sci. Rev. 2014, 50, 69-77. [CrossRef]

16. Bizhang, M.; Schmidt, I.; Chun, Y.-H.P.; Arnold, W.H.; Zimmer, S. Toothbrush abrasivity in a long-term simulation on human dentin depends on brushing mode and bristle arrangement. PLoS ONE 2017, 12, e0172060. [CrossRef] [PubMed]

17. Spina, V. A proposed modification for the classification of cleft lip and cleft palate. Cleft Palate J. 1973, 10, 251-252. [PubMed]

18. Dentistry, A.A.O.P. Guideline on periodicity of examination, preventive dental services, anticipatory guidance/counseling, and oral treatment for infants, children, and adolescents. Pediatr. Dent. 2013, 35, E148.

19. Greene, J.C.; Vermillion, J.R. The simplified oral hygiene index. J. Am. Dent. Assoc. 1964, 68, 7-13. [CrossRef] [PubMed]

20. Ashley, P. Toothbrushing: Why, when and how? Dent. Update 2001, 28, 36-40. [CrossRef] [PubMed]

(C) 2018 by the authors. Licensee MDPI, Basel, Switzerland. This article is an open access article distributed under the terms and conditions of the Creative Commons Attribution (CC BY) license (http://creativecommons.org/licenses/by/4.0/). 